\title{
Derechos humanos del adulto mayor en el ámbito familiar colombiano en el marco del envejecimiento demográfico
}

\section{Aging demographics, familie and the elderly in comtemporary culture}

\author{
DOI: https://doi.org/10.17981/juridcuc.16.1.2020.09
}

Fecha de Recepción: 12/12/2019. Fecha de Aceptación: 03/02/2020

\author{
Olga Suárez Landazábal \\ Universidad Metropolitana (Colombia) \\ olga.suarez@unimetro.edu.co
}

\section{Francis Araque Barboza \\ Universidad Metropolitana (Colombia) tita42_fa@yahoo.es}

Para citar este artículo:

Suárez, O. y Araque, F. (2020). Derechos humanos del adulto mayor en el ámbito familiar colombiano en el marco del envejecimiento demográfico. Jurídicas CUC, 16(1), 225-250. DOI: http://dx.doi.org/10.17981/juridcuc.16.1.2020.09

\section{Resumen}

El artículo reseña los cambios en la familia contemporánea, al analizar el impacto del aumento demográfico de los adultos mayores en la organización familiar. Se fundamenta en una disertación temática narrativa de postulados teóricos nacionales e internacionales sobre las manifestaciones familiares y de sus miembros a partir de una metodología documental-bibliográfica. Las perspectivas epistémicas aquí reunidas tejen puntos de encuentros entre el derecho, la demografía, y la sociología, con las grandes tendencias del cambio poblacional. Se evidencia la existencia de una tensión entre las necesidades del adulto mayor y sus derechos inalienables en un entramado familiar afectado por la ausencia de una ética para el bien común compartida. Se promueve desde la perspectiva axiológica los derechos humanos del adulto mayor en el ámbito de la vida familiar.

Palabras clave: Adulto mayor; familias; envejecimiento demográfico; derechos humanos

\begin{abstract}
The article reviews the changes in the contemporary family by analyzing the impact of the demographic increase of the elderly on the family organization. It is based on a thematic narrative dissertation of national and international theoretical postulates on family manifestations and their members based on a documentary-bibliographic methodology. The epistemic perspectives gathered here weave points of encounter between law, demography, and sociology, with the great tendencies of population change. There is evidence of a tension between the needs of the elderly and their inalienable rights in a family network affected by the absence of an ethic for the shared common good. The human rights of the elderly in the area of family life are promoted from an axiological perspective.
\end{abstract}

Keywords: Elderly; families; demographic aging; human rights 


\section{INTRODUCCIÓN}

La familia como hecho social ha generado numerosos estudios, los cuales enriquecen lo que hoy se conoce acerca del tema, genéricamente define los procesos de socialización y de protección psicoafectiva de sus miembros, sin embargo, estos se encuentran mediados por dinámicas económicas, poblacionales, políticas e ideológicas, tales como la incorporación de la mujer al mercado laboral, los cambios demográficos, el desplazamiento forzado, las migraciones, el impacto de las TICS (Tecnologías de Información y Comunicación) y los divorcios, entre otros. El objetivo de este artículo es analizar sobre el adulto mayor, presente en el espacio familiar y sus derechos humanos a fin de promover desde un enfoque axiológico el respecto al otro, paseando la mirada epistémica de lo estrictamente biomédico a términos sociopolíticos que denuncien la desigualdad mediada por lo económico y afectivo en una sociedad donde los valores humanos se encuentran ampliamente cuestionados. De hecho, la CEPAL (Comisión Económica para América Latina y el Caribe) al analizar la familia, sus condiciones, organización, estructura y evolución plantea la existencia de dificultades para alcanzar el ejercicio pleno y efectivo de los derechos humanos de todos sus miembros sin importar su edad y género.

Desde la perspectiva asumida en este trabajo, las familias son realidades no homogéneas, con lógicas de comportamientos diversas, donde cada uno de sus miembros reciben de diferente manera los elementos que tocan y trastocan el funcionamiento familiar, factores tales como cambio en su estructura, aumento del envejecimiento demográfico, aumento del divorcio, entre otros, son una realidad. De allí que no exista una forma única de familia, haciendo que el primer elemento a tomar en cuenta para la discusión de las familias con miembros adultos mayores y su derecho a la inclusión social es su polimorfismo, triangulares, monoparentales, reestructuradas, recompuestas, ampliadas u otras aclaman en las últimas décadas existe la necesidad de articular el derecho en la compleja realidad familiar actual, las normas jurídicas deben ser integrales, 
holísticas y circulares mediante leyes de reconocimientos donde las nuevas familias, tocadas por el aumento de la expectativa de vida de todos sus miembros merecen respeto. Es importante construir un discurso propio de esta época donde la primera obligación que debe presentarse en cualquier familia es la de la alimentación de sus integrantes. En Colombia, donde aproximadamente el $70 \%$ de los adultos mayores no tienen pensión y $22 \%$ viven en la pobreza, es deber del Estado impulsar a las familias legalmente a proteger y atender a las personas que se encuentran en los años dorados (Farné y Rodríguez, 2014)

Finalmente, la finalidad de esta propuesta propicia la colaboración interdisciplinar de la demografía, la sociología y las ciencias jurídicas, para instaurar un escenario de reflexión consensuada e inclusión epistemológica con sus diferentes aportes en el estudio propio de las familias en la contemporaneidad conformadas por miembros de la tercera edad.

\section{Envejecimiento demográfico e impacto en la estructura familiar}

El envejecimiento demográfico es un proceso que se ha extendido a nivel mundial haciendo visible cada vez más a los adultos mayores, por el aumento significativo de esta población frente a los demás grupos etarios debido al aumento de la esperanza de vida.

Para ser considerado adulto mayor implica llegar a la edad de 60 años para países en vía de desarrollo como Colombia, o a los 65 años en países desarrollados y es el inicio de la denominada etapa de la vejez, donde se evidencian más fuertemente los cambios en el organismo y surgen, por tanto, necesidades y situaciones que implican el apoyo incondicional de la familia como primera red de soporte social.

Para la Organización Mundial de la Salud (OMS, 2017), "por primera vez en la historia, la mayoría de las personas pueden esperar vivir hasta los 70 años e incluso más" (p. 1). La población mundial de más de 60 años pasará entre el 2015 y 2050, de 900 millones hasta 2000 millones, lo cual representa un aumento del $12 \%$ al $22 \%$. 
Según la Organización de las Naciones Unidas (DAES, 2017), la expectativa de vida ha aumentado a nivel mundial, al ser para los años 2000 a 2005 de 65 años para los hombres y 69 para las mujeres. Entre 2010 y 2015 , los hombres alcanzaron una edad de 69 años y las mujeres de 73 años.

A nivel mundial, entre el 2000 y el 2050 la cantidad de personas de 80 años o más aumentará casi cuatro veces hasta alcanzar los 395 millones. Es un acontecimiento sin precedentes en la historia que la mayoría de las personas de edad madura e incluso mayores tengan unos padres vivos, como ya ocurre en nuestros días. Ello significa que una cantidad mayor de los niños conocerán a sus abuelos e incluso sus bisabuelos, en especial sus bisabuelas. En efecto, las mujeres viven por término medio entre 6 y 8 años más que los hombres (OMS, 2019).

Las razones del envejecimiento demográfico se relacionan con el desarrollo científico y tecnológico, el mejoramiento de las condiciones de salud de la población que se revierten en el aumento de la expectativa de vida e igualmente por los descensos de la fecundidad y de la mortalidad (Ministerio de Salud y Protección Social-Minsalud, 2013).

Según CELADE (Centro Latinoamericano y Caribeño de Demografía), en América Latina y el Caribe, hacia el año 2040 el porcentaje de personas de 60 años o más superará por primera vez a los menores de 15 años, lo que supondrá una nueva estructura por edad. La tasa de fecundidad pasó aceleradamente de 6 a 3 hijos por mujer en 25 años y actualmente, el promedio de la región está por debajo de la tasa de reemplazo que alcanza a los 2,1 hijos por mujer. Para el año 2014, la esperanza de vida al nacer en América Latina fue de 74.8 años, la que se estima que aumentará a 81.6 en el período 2045-2050 (CEPAL, 2017).

Para el caso de Colombia, en el año 2015, existían 5,2 millones de personas mayores de 60 años que correspondían al 10,8\%, para el año 2030 se estima que será un 18,3\% y para el 2050 llegará a un 27,6\% (Helpage International, 2015). Los datos del censo del 2018 indican que la población de mayores de 60 años sigue en aumento 
donde el 13,3\% están en este rango de edad (Departamento Administrativo Nacional de Estadística, DANE, 2018).

Por otra parte, el envejecimiento demográfico muestra diferencias entre hombres y mujeres, donde los índices de masculinidad para el año 2010, descendieron progresivamente a lo largo del ciclo de vida incrementándose la proporción de mujeres al pasar de 104 hombres por cada 100 mujeres en el grupo de los menores de 15 años, a 73 hombres por cada 100 mujeres en el grupo de 80 y más años de edad (Minsalud, 2013).

Otro aspecto a destacar y causante del envejecimiento demográfico hace referencia a la reducción de la fecundidad, la cual en Colombia se inició en la segunda mitad de la década de los 60 , con un descenso que se mantuvo muy intenso hasta mediados de la década de los 80 , convirtiéndose entre los más acentuados en el contexto de América Latina. Este descenso ha continuado a través de los años con menor intensidad y ha llegado a una tasa global de fecundidad para el año 2015 de 2 hijos por mujer, con una diferencia entre la población urbana (1.8 hijos/mujer) y la rural (2.6 hijos/mujer). Además, el porcentaje de mujeres y hombres en edad fértil sin hijos e hijas es un indicador tan importante como el promedio de hijos e hijas nacidos vivos, donde el 35.6\% de las mujeres y el 52.7\% de los hombres de 13 a 49 años no tienen hijos e hijas (Minsalud, 2015).

La disminución de la tasa de fecundidad definitivamente tiene su influencia en el tamaño, composición y funcionamiento de los hogares. En Colombia, al igual que en América Latina, se ha visto un descenso de los hogares con familias extendidas y compuestas. Aumento de las parejas sin hijos y de los hogares sin núcleo conyugal; incremento de los hogares monoparentales, especialmente los de jefatura femenina, y aumento de arreglos de convivencia que no incluyen parentesco. Todo esto ha llevado a un marcado descenso del tamaño de los hogares, siendo para el año 2015 de 3,5 personas por hogar. Por otra parte, la jefatura femenina es un fenómeno que viene en incremento mostrando para el 2015 un promedio nacional de 36.4 por ciento de los hogares que declaran a una mujer como jefe del hogar (Minsalud, 2015). 
El último censo llevado a cabo en Colombia en el 2018 evidenció que el promedio de personas por hogar es de 3,1. Por tanto, siguen disminuyendo los integrantes de las familias. En cuanto a la jefatura femenina, esta sigue en aumento con un 40,7\%, mientras que en el censo del 2005 estaba en 29,9\% (DANE, 2018).

El hecho de mayor expectativa de vida y el descenso de la fecundidad llevan a desarrollar familias con más generaciones viviendo a la vez, pero menos extensas, es decir, con menos generaciones colaterales y se observan cifras de personas parecidas entre abuelos, hijos y nietos, este proceso se denomina verticalización de la familia. Esta conformación de la familia lleva a que se establezcan más relaciones "verticales" que antes, y se den entonces tareas de más responsabilidad o transferencias desde abajo hacia arriba o viceversa en esta escala vertical familiar (Abellán \& Esparza, 2009). Una de las consecuencias de esta situación es la posibilidad de generar familiares que ameriten cuidados, necesitando entonces, una reorganización familiar para cumplir con esta realidad donde se cuentan con pocos integrantes.

Los cambios producto del envejecimiento demográfico definitivamente han tenido una incidencia significativa en las transformaciones de la familia. Si se analiza el hecho del aumento de la expectativa de vida significa que se extiende la etapa de la vejez. Este hecho se convierte en un fenómeno del cual muchas instituciones no han tomado conciencia de su magnitud y celeridad. Por tanto, la familia deja de lado su esencia autónoma como forma social autosuficiente y se hace permeable a las nuevas configuraciones que se visualizan en su estructura (Landriel, 2001).

La familia es un sistema de interrelación biopsicosocial que media entre el individuo y la sociedad y se encuentra integrada por un número variable de individuos, unidos por vínculos de consanguinidad, unión, matrimonio o adopción (Torres, Ortega, Garrido y Reyes, 2008, p. 32).

Desde el aspecto Sociológico, se considera como un grupo de personas entrelazadas en un sistema social, cuyos vínculos se basan 
en relaciones de parentesco fundados en lazos biológicos y sociales con funciones específicas para cada uno de sus miembros y con una función más o menos determinada en un sistema social (Cadavid, 1994, p. 331).

Desde una visión religiosa la familia se encuentra constituida por la trinidad (Padre, Hijo y Espíritu Santo), comprendiendo que una sola de ellas no podría dar a entender la imagen de Dios (Sigüenza, 2015, p. 16).

Desde el punto de vista psicológico, se "define como un grupo primario, como un grupo de convivencia intergeneracional, con relaciones de parentesco y con una experiencia de intimidad que se extiende en el tiempo" (Maxler y Mishler citado por Gallego, 2012, p. 331).

Desde el punto de vista jurídico, la Constitución Política de Colombia (Const., 1991, art. 42), define la familia como "el núcleo fundamental de la sociedad, constituida por vínculos naturales o jurídicos, por la decisión libre de un hombre y una mujer de contraer matrimonio o por la voluntad responsable de conformarla". También es reconocida por ésta como la institución más importante y el núcleo de la sociedad, así, se encuentra protegida constitucional y legalmente.

Para Munichin (citado por Gallego, 2012) la familia se define como "el conjunto invisible de demandas funcionales que organizan los modos en que interactúan los miembros de una familia y que las relaciones e interacciones de los integrantes están condicionadas a determinadas reglas de comportamiento" (p. 331).

Intrínsecamente la familia está determinada por una estructura, un subsistema (conyugal, parental y fraternal), etapas del ciclo vital familiar, valores y creencias, hábitos, costumbres y dificultades relacionadas con la salud.

Las tipologías familiares pueden ser varias, en algunos casos según los vínculos existentes entre sus miembros, los cuales pueden ser consanguíneos, por afinidad, adopción, o amistad. También la convivencia es un elemento importante en este concepto porque las familias se clasifican a partir de las personas que comparten el 
mismo techo, lo que le confiere a la familia unos rasgos o caracteres peculiares (Agudelo, 2005, p. 7).

Araque y Rodríguez (2008) describen la tipología familiar de una forma muy concisa, la familia unipersonal, como su nombre bien lo indica, es aquella en donde vive una solo personal que generalmente es un adulto.

La familia monoparental femenina y monoparental masculina "es aquella conformada por un solo cónyuge y sus hijos, surge como consecuencia del abandono del hogar de uno de los cónyuges, por fallecimiento, separación y/o divorcio, madresolterismo o padresolterismo" (Araque y Rodríguez, 2008, p. 7)

Las uniones consensuales, son aquellas unidades familiares en las cuales hay comunidad de hechos y techos, asidua y duradera y, en donde la vida en parejas se desarrolla ostensiblemente a los ojos de los demás, como si se tratara de un verdadero matrimonio (Araque y Rodríguez, 2008, p. 7)

La Familia adoptiva, según Araque y Rodríguez (2008) "nace de acto jurídico o resolución judicial y que crea entre dos personas, que no son necesariamente parientes consanguíneos, relaciones ficticias y civiles de parentesco y filiación" (p. 31)

La unidad domestica u hogar colectivo, es una estrategia de sobrevivencia donde las personas están unidas voluntariamente para aumentar el número de perceptores económicos y lograr economías de escala. Algunas formas de hogar más reconocidas son cuarteles, conventos, campamentos, asilos, hospitales, hogares estudiantiles, cohabitación juvenil, etc. Como experiencias de convivencia, se registran también los hogares de niños de la calle, orfelinatos $u$ hogares sustitutos, entre otros (Rodríguez, Araque y Salazar, 2009, p. 96).

La familia simultánea o agregada, donde uno o ambos cónyuges tienen hijos de relaciones anteriores y en la actualidad la pareja convive con estos y/o con los hijos de ambos. "Esta es conocida comúnmente como 'tus hijos, mis hijos y los nuestros', es aquella donde llega un nuevo miembro a asumir un papel sustituto debido a la ausencia del progenitor biológico (Agudelo, 2005, p. 7). 
Las familias actuales cuentan con menos integrantes y tiene menos posibilidades de acompañamiento para los adultos mayores, haciéndolos susceptibles a la soledad y abandono. Sin embargo, tal como lo expresa Lehr (1995), la familia no debe ser considerada como una entidad estática, sino al contrario como una entidad que vive un proceso dinámico interactivo a lo largo de la vida, debido a que la evolución y cambios que afectan a un miembro de la familia conllevan a su vez evolución y cambios en cada uno de los restantes miembros. Solo con esta concepción dinámica se podrá abordar de una forma adecuada la relación entre los miembros de las distintas generaciones.

De acuerdo a todos estos cambios en la familia, Pedroza, SuárezLandazábal y Vargas (2018) expresan que las nuevas generaciones a nivel mundial muestran una de las más grandes preocupaciones y es cómo afrontar el periodo de la vejez, una etapa la cual en el pasado se asumía con total naturalidad, pero actualmente constituye un tema dilémico, considerando los cambios en los valores generados a partir de procesos económicos, políticos, sociales, culturales y familiares.

\section{Familia, derechos y cuidado del adulto mayor}

El envejecimiento humano como proceso significa un cambio en todos los sistemas del cuerpo que se traducen en disminución de todas sus funciones, y esta disminución necesariamente es más evidente en la etapa de vejez, por tanto, vivir más años no significa vivir con suficiente funcionalidad para todos los adultos mayores. El impacto de la edad deja en evidencia que las personas de entre 65 y 74 años tienen considerablemente menos necesidad de ayuda que las personas de 75 años o más (OMS, 2015).

Si se habla de la funcionalidad, se hace referencia al mantenimiento de la independencia en los aspectos físico, mental, sociofamiliar y económico (Pedrero \& Pichardo, 2009), por tanto, cuando el cuerpo y la mente son capaces de llevar a cabo las actividades de la vida cotidiana se dice que la capacidad funcional está indemne (Segovia y Torres, 2011, p. 63). 
Según Suárez-Landazábal, Batista y Bornacelli (2018), es pertinente evitar llegar a la situación de dependencia en la etapa de la vejez, tan temida por el ser humano, ya que esta condición no solo afecta a la persona, sino también a quienes conviven con ella, debido a que les genera cambios de orden social, económico, psicológico entre otros. Al respecto Suárez-Landazábal (2018) destaca el valor del medio ambiente y de los hábitos saludables a lo largo de la vida tales como actividad física y alimentación, dada la fuerte influencia que tienen en el envejecimiento saludable.

Se pronostica que de aquí al año 2050 la cantidad de ancianos que no pueden valerse por sí mismos se multiplicará por cuatro en los países en desarrollo. Muchos ancianos de edad muy avanzada pierden la capacidad de vivir independientemente porque padecen limitaciones de la movilidad, fragilidad u otros problemas físicos o mentales. Muchos necesitan alguna forma de asistencia a largo plazo, que puede consistir en cuidados domiciliarios o comunitarios y ayuda para la vida cotidiana, reclusión en asilos y estadías prolongadas en hospitales (OMS, 2019).

El cambio demográfico hará más visible la discapacidad que es el resultado no expresamente del envejecimiento sino también del estilo de vida que se haya tenido a lo largo del proceso de envejecimiento y de ahí las cifras alarmantes de enfermedades crónicas no transmisibles tales como la hipertensión arterial, la diabetes, las enfermedades cardiovasculares entre otras.

Según la OMS (2011) en el mundo hay más de 1000 millones de personas con discapacidad, lo que supone alrededor de un 15\% de la población mundial, o una de cada siete personas. Y para América Latina y el Caribe el porcentaje de adultos mayores con discapacidad de acuerdo a los censos de 2010 para 8 países latinoamericanos (Brasil, Costa Rica, Ecuador, México, Panamá, República Dominicana, Uruguay y Venezuela), mostró que aproximadamente el 41\% de las personas mayores tenían algún tipo de discapacidad. Se destaca Brasil (más del 60\%), así como República Dominicana y Uruguay (45\%). La incidencia es más elevada en las mujeres que en los hombres: un $43 \%$ frente a un $38,8 \%$. De acuerdo a los censos del 2010, se 
resalta el hecho de que las mujeres registran una tasa de prevalencia de discapacidad más alta que la de los hombres, debido a que su mayor esperanza de vida aumenta la posibilidad de que tengan una discapacidad asociada a una enfermedad crónica o un accidente en etapas avanzadas de la vida (Huenchuan, 2018).

Colombia no tiene una cifra exacta de personas con discapacidad, no obstante, el Censo del DANE de 2005 captó a 2.624 .898 (6,3\%) personas que refirieron tener alguna discapacidad. Desde el año 2.002 a través del Registro de Localización y Caracterización de Personas con Discapacidad (RLCPD) se han identificado a junio del 2018, 1.404 .118 personas, que corresponden al 2.6\% de la población total. Según este registro, el 58\% (809.853) de las personas son mayores de 50 años de edad. Se destaca igualmente que, de 100 personas mayores de 80 años, 33 están en el registro de discapacidad y a partir de los 50 años, es mayor la proporción de mujeres con discapacidad frente a los hombres (Minsalud, 2018).

Los reportes del RLCPD, muestran como el movimiento del cuerpo es la alteración que más afecta a las personas con discapacidad, referido por el 34\% (470.215), seguido del sistema nervioso en un $23 \%$ (350.216), el 13\% (179.471) los ojos, le siguen el sistema cardiorespiratorio y las defensas en un $10.3 \%$. Con relación a la población adulta mayor, la alteración que más afecta es el movimiento del cuerpo en un 41\% (215.989), seguida del sistema cardiorrespiratorio en un 16\% (86.536). Además, el 50\% de las personas con discapacidad presentan dificultad para caminar, correr y saltar, el 38\% dificultad para pensar y memorizar, $22 \%$ para desplazarse en trechos cortos (Minsalud, 2018).

El censo del 2018 en Colombia, corrobora que mover el cuerpo, caminar o subir y bajar escaleras es la dificultad de mayor prevalencia con un $32 \%$ y le siguen la dificultad para ver de cerca, de lejos y alrededor con un $19 \%$ y para oír la voz o los sonidos con un $18 \%$. Así mismo, la prevalencia de personas con alguna dificultad para realizar actividades diarias de acuerdo al sexo muestra un predominio en las mujeres con 7,5\% frente a los hombres con un 6,8\% (DANE, 2018). 
Con el aumento de la edad aumenta también la dependencia y se hacen necesarios los cuidados, es así como el envejecimiento demográfico aumentará significativamente la población que necesite de asistencia de diferente índole en todos los países. A esto se suma que la proporción de personas más jóvenes que podría proporcionar esa atención seguirá disminuyendo, y al tiempo, el papel de las mujeres ha venido cambiando, dejando su función de ser proveedoras de atención. Además, en muchos países con mayor necesidad tienen a su vez menor infraestructura y servicios para hacer frente a la dependencia de cuidados, dejando esta responsabilidad a las familias (OMS, 2015). Pero como se ha visto la estructura familiar ha cambiado afectando las relaciones familiares y la forma como debe responder ante el evento de una enfermedad o perdida de independencia de los adultos mayores.

La familia que tiene a su cuidado un adulto mayor dependiente, enfrenta cambios de diferente índole, por una parte, esta situación de cuidar, por lo general es de largo tiempo, además presentará dificultades de convivencia por los conflictos que surgen sobre quién debe hacerse cargo del adulto mayor, se harán necesarias adaptaciones del espacio físico y lógicamente cambios en el aspecto económico de la familia.

En Colombia, según la encuesta SABE 2015, con relación a los cuidadores de adultos mayores, 83.9\% eran mujeres. Por lo regular, $61 \%$ son hijos de las personas mayores que cuidan, 10\% cónyuges, $14 \%$ nietos, padres o hermanos y menor al 10\% la familia política u otras personas (Minsalud y Colciencias, 2016). El cuidado de los hijos hacia los padres es el mejor ejemplo de solidaridad, pero si analizamos que cada vez son menos los hijos disponibles para tal función, se puede convertir en una tarea difícil de llevar a cabo por el desgaste que significa y por el deterioro de las relaciones familiares, donde la mayor carga es asumida principalmente por las mujeres.

De acuerdo a estas cifras, el cuidado recae prácticamente sobre la familia del adulto mayor como fuente más importante del cuidado en la vejez. 
Los cuidados que con mayor frecuencia se realizan tienen que ver con las actividades instrumentales de la vida diaria, como administrar medicamentos, salir a la calle, hacer compras e ir al médico. Solamente el 12,5\% prestaba cuidados especializados como manejo de sondas, equipos médicos o terapias específicas. Dos terceras partes de las personas cuidadoras familiares manifestaron que realizan las labores de cuidado por iniciativa propia. El tiempo dedicado para el cuidado oscila entre 1 y 24 horas, la mitad de ellos dedicó 10 horas o menos diariamente a las labores de cuidado; la mayoría de las personas cuidadoras (87.2\%) realizaron las actividades del cuidado todos los días, lo cual implica ausencia de tiempo libre o de ocio y conduce a sobrecarga, debido a que se trata de una labor continuada que perdura con el tiempo, que impide cuidar de sí mismos (Minsalud y Colciencias, 2016).

Es necesario reflexionar alrededor de los cambios a nivel demográfico reflejados en la familia, los cuales llevan a que haya menos personas disponibles para cuidar, poniendo en riesgo ese deseo tan anhelado por todos de envejecer en casa, que es el ideal y debe ser reforzado con políticas sociales apoyando en este sentido.

Por otra parte, se puede presentar otra situación, en la cual, aunque en la familia hayan reducido los hijos y los nietos, el hecho de que la expectativa de vida se haya ampliado, permite en ciertas familias a las personas mayores conservar a sus padres, dejando ver la posibilidad del cuidado de abuelos y bisabuelos, es decir, adultos mayores cuidando a otros adultos mayores.

Ante la realidad expuesta de los cambios sociodemográficos que vive el mundo, las familias deberían estar preparadas y sensibilizadas para la convivencia y atención de sus adultos mayores desde diferentes perspectivas biopsico-sociales y jurídicas, como es el conocimiento del proceso de envejecimiento para comprender los cambios que trae la vejez, o las adaptaciones de espacios, entre otros, con el fin de plantear iniciativas que mejoren las condiciones de los adultos mayores. Así como también la necesidad de comprender las normas jurídicas integradas a los nuevos arreglos familiares, mediante leyes de reconocimientos que estimulen la redistribuccion y los acuerdos cooperativos entre sus miembros (Nieves y Maldonado, 2011, p. 18). 
Dado que las organizaciones familiares emergentes en la contemporaneidad no son vinculantes en el derecho, pero si sancionadas socialmente y favorecidas casuísticamente por las sentencias de la honorable Corte Constitucional colombiana, es imprescindible revisar el orden jurídico en este artículo. Para Pulido, Calderón y Arteaga (2018) en Colombia aún se comprende a las familias como un sistema estático, donde existe un horizonte y unas metas predeterminadas, siendo percibidas desde una hegemonía estructurada en límites y restricciones; lo cual se puede evidenciar en la Constitución Política de Colombia donde define la familia como núcleo primordial de esta sociedad (Const., 1991, art. 42), lo cual conlleva a la deslegitimación de la modificaciones sociales, emocionales y culturales, y a su vez, no se tiene en cuenta la diversidad para definir a la familia.

Igualmente, la Ley 1251 (2008), donde se dictan normas tendientes a procurar la protección, promoción y defensa de los derechos de los adultos mayores y plantea de parte del Estado algunos de los deberes tales como "proteger y restablecer los derechos de los adultos mayores cuando estos han sido vulnerados o menguados, promover una cultura de solidaridad hacia el adulto mayor; eliminar toda forma de discriminación, maltrato, abuso y violencia sobre los adultos mayores. Desde la Familia también están como deberes el de propiciar un ambiente de amor, respeto, reconocimiento y ayuda; brindar un entorno que satisfaga las necesidades básicas que mantengan una adecuada nutrición, salud, desarrollo físico, psíquico, psicomotor, emocional y afectivo; brindar apoyo y ayuda especial al adulto mayor en estado de discapacidad entre otros.

Por otro lado, para el año 2014 se realizó un informe por parte de las instituciones y universidades de distintos países llamado World Family Map (Child Trends, 2014), el cual permite distinguir 16 indicadores que identifican el estado social, económico, educativo y político de las familias, siendo estos: la pobreza como un factor determinante en relación al desarrollo y futuro avance de una familia, y a su vez, no afecta de manera igual a todos los miembros del grupo familiar, siendo los más, endebles las mujeres, los adultos mayores y los niños, esta realidad a largo plazo trae limitaciones que determinan el poco 
impacto de las políticas públicas que ejecuta el Estado a favor de la familia.

Pachón (2008) refiere cual es la situación que tienen las familias colombianas a lo largo de la historia y el presente, mencionando:

- La estructura familiar en Colombia suele ser idealizada por su población, lo cual ocasiona dificultades, como la violencia oculta y se acepta hechos simbólicos que irían en contra de la vida de los sujetos que hace parte de la sociedad.

- A inicios del siglo XX, la familia colombiana tenía más marcado un pensamiento conservador y patriarcalista, trayendo consigo una serie de retos para los miembros de la familia, entre los cuales se resaltan la violencia contra la mujer, el abandono de los hijos que no nacían dentro de un matrimonio y la división de las familias extensas.

- A mitad del siglo XX, influyeron varios factores que provenían del conflicto interno que ha vivido el país, entre los cuales está la violencia armada y el desplazamiento, ocasionando a que las familias en Colombia generen un cambio en sus dinámicas, desde la perspectiva ideológica familiar como en el sentir conservador que marcaba la época.

- En los años 70 y 80 se rompe la estructura patriarcal, permitiendo que se realicen de manera significativa una modificación de las relaciones jerárquicas, y a su vez, se genera un debilitamiento en la influencia eclesiástica en relación a las familias colombianas. Así mismo, se evidencia que se da un avance significativo en los derechos del niño y la mujer, ocasionando a que se promueva una cultura más democrática.

- A finales del siglo XX e inicios del siglo XXI, se refleja una mayor autonomía en las familias en Colombia, en cuanto a la estructura familiar y su relación con las ideologías dominantes, lo cual se evidencia en la disminución de los matrimonios en relación a épocas anteriores; así mismo, se generan nuevas relaciones que conllevan a que se modifique la estructura al interior de las familias, entre las cuales se pueden mencionar la unión de parejas del mismo sexo y el monoparentalismo. 
Es así, como se puede observar como a lo largo de la historia la concepción que se tiene de las familias colombianas, ha estado influenciada por los acontecimientos que han sucedido en distintas épocas, y que conllevan a que se replante la definición de familia no solo desde el ámbito jurídico sino también desde imaginario social, para no deslegitimar a aquellas personas que son miembros de familias diferentes a la familia nuclear.

Teniendo en cuenta que la Constitución Política de Colombia (1991), señala:

Artículo 5. El Estado reconoce, sin discriminación alguna, la primacía de los derechos inalienables de la persona y ampara a la familia como institución básica de la sociedad.

Artículo 13. Todas las personas nacen libres e iguales ante la ley, recibirán la misma protección y trato de las autoridades y gozarán de los mismos derechos, libertades y oportunidades sin ninguna discriminación por razones de sexo, raza, origen nacional o familiar, lengua, religión, opinión política o filosófica. El Estado promoverá las condiciones para que la igualdad sea real y efectiva y adoptará medidas en favor de grupos discriminados o marginados. El Estado protegerá especialmente a aquellas personas que, por su condición económica, física o mental, se encuentren en circunstancia de debilidad manifiesta y sancionará los abusos o maltratos que contra ellas se cometan.

Artículo 15. Todas las personas tienen derecho a su intimidad personal y familiar y a su buen nombre, y el Estado debe respetarlos y hacerlos respetar. De igual modo, tienen derecho a conocer, actualizar y rectificar las informaciones que se hayan recogido sobre ellas en bancos de datos y en archivos de entidades públicas y privadas.

Artículo 28. Toda persona es libre. Nadie puede ser molestado en su persona o familia, ni reducido a prisión o arresto, ni detenido, ni su domicilio registrado, sino en virtud de mandamiento escrito de autoridad judicial competente, con las formalidades legales y por motivo previamente definido en la ley. 


\section{Adultos mayores viviendo solos: ¿Dónde quedó la familia?}

El envejecimiento demográfico está favoreciendo los hogares unipersonales llevando a una tendencia a vivir solo a nivel global: entre 1996 y 2011 el número de personas que viven solas en el mundo pasó de 153 a 277 millones. Esta realidad es el resultado del descenso de la tasa de fecundidad, aumento de la esperanza de vida, con el proceso de la individuación de las sociedades, con la expansión de los sistemas de seguridad social y con otros cambios culturales entre los cuales está nuevas formas de organización de la familia y lógicamente con el hecho de la incursión de la mujer al ámbito laboral (Jaramillo, 2019).

La soledad es uno de los males que acecha a la sociedad contemporánea, a tal punto que en Reino Unido se volvió un asunto de Estado. Se estima que, en Inglaterra, la mitad de los ancianos de 75 años viven solos, lo que equivale a unos 2 millones de personas. Muchos de ellos dicen que pasan días, incluso semanas, sin ningún tipo de interacción social. La primera ministra británica, Theresa May, anunció esta semana la creación de un Ministerio de la Soledad (BBC Mundo, 2018).

En América Latina y el Caribe desde 1990 el porcentaje de hogares de personas mayores que viven solas ha venido en aumento. Para este año, la proporción no superaba el 4\%, a excepción de Argentina y Uruguay que presentaban valores cercanos al 7\% y 10\% respectivamente. Para el año 2000, el porcentaje mostró una tendencia a aumentar, pero los valores siguen siendo bajos. Para los censos de la década del 2010 se observó un notorio aumento en los países estudiados (Argentina, Bolivia, Brasil, Chile, Costa Rica, Cuba, Ecuador, Honduras, México, Panamá, República Dominicana, Uruguay y Venezuela). Los valores más altos se registran donde el envejecimiento demográfico está más avanzado: Uruguay, Argentina, Cuba y Chile (Huenchuan, 2018).

Si se mira el peso relativo de los adultos mayores que viven solos en el total de la población igualmente se observa que este ha aumentado en todos los países: El 23\% de las personas mayores 
del Uruguay viven solas, y en la Argentina y Bolivia el porcentaje supera el $15 \%$, mientras que en el resto de los países rebasa el 10\%, a excepción de Honduras, donde alcanza el 7\% (Huenchuan, 2018).

Para el caso de Colombia, los hogares unipersonales han venido en aumento, ya que en el censo del 2005 representaban el 11,1\%, mientras que en el censo del 2018 fue de 18,1\%, reflejando una tendencia de los colombianos a vivir solos (DANE, 2018).

Este cambio en Colombia en la conformación de hogar unipersonal se dio en forma acelerada (entre 50 y 80 años). Dichos hogares unipersonales no están tan igualmente concentrados en las ciudades y no disfrutan de independencia física y económica. La realidad muestra que tanto en las zonas rurales como en las ciudades del país estos hogares generalmente se encuentran en condición de pobreza. Esta situación se relaciona con la proporción de adultos mayores pensionados, ya que, de cada 10 mayores de 60 años, apenas dos reciben pensión, es decir, que otros ocho dependen sobre todo de familiares, amigos o vecinos. Esto afecta el bienestar de los adultos mayores y, por ende, el de sus familiares, quienes deben asumir las funciones de protección que debería asumir el Estado (Jaramillo, 2019).

La situación del hogar unipersonal aumenta según se avanza en edad y puede surgir cuando uno de los cónyuges fallece, pero cambia cuando aparece la discapacidad y dependencia impidiendo seguir de esta forma.

Sin embargo, otra forma de ver la opción del hogar unipersonal según la encuesta SABE 2015 Colombia (Minsalud y Colciencias, 2016), es considerándola una expresión de calidad de vida y en un aporte a la autonomía e independencia, en contravía al imaginario de la soledad, el abandono familiar y la existencia de débiles vínculos afectivos.

Para mejorar las condiciones de vida de los adultos mayores hay que ampliar la cobertura y el acceso a la pensión, fortalecer las redes sociales y familiares con el fin de favorecer la compañía constante de este grupo etario. 
El hecho de que los adultos mayores vivan solos, no significa que no tengan vínculos familiares, ya que la familia es la red primaria de soporte social y recurren a ella ante calamidades, para tomar decisiones, para celebrar fechas y ante eventos que se relacionen con recursos económicos. Otra red de soporte son las amistades y los vecinos con quienes comparten diferentes situaciones tanto de tipo material como emocional. Aquí también se considera la tecnología virtual para mantener la comunicación con la familia como son el facebook y whatsApp (Minsalud y Colciencias, 2016).

\section{Conclusiones}

Según Cerruti y Bintock (2009), en las últimas décadas en América Latina se han suscitado una serie de transformaciones sociales, demográficas, económicas, entre otras, que han afectado las dinámicas de la vida familiar. Estos cambios han alterado, las relaciones estructuras, relaciones, los cuales han exigido y orientado desde el derecho las vivencias y significaciones de las familias al exigir un trato más humano y correcto con todos sus integrantes.

En una sociedad se debe considerar que un acto correcto no es aquel que lleva a la felicidad general es el correcto para todos, un acto justo que contemple los principios universales que son todos, que regulan la estructura base de la sociedad económica y política y unos principios públicos que son aquellos que todo ciudadano debe tener y conocer (Gargarella, 2019).

En efecto, según Reales (2016), la existencia de democracia no garantiza la preservación de los derechos humanos. Los países subdesarrollados como Colombia, cuando no tienen una estructura orgánica que acredite una solidez en sus instituciones no satisfacen plenamente las necesidades de sus asociados por nimias que sean las reivindicaciones, entonces, acude a expedientes dilatadores que soslayan la médula de las obligaciones imperativas. Tal como expresan Hernández y Chumaceiro (2018), la generalidad de la región ha expresado separación entre ejercicio democrático 
y el pleno reconocimiento de la ciudadanía y en específico de sus derechos. De hecho, para que violen los derechos no hace falta que estalle un petardo ó que explote un carro bomba, sólo basta con la desatención alimentaria a nuestro parecer de los grupos más vulnerables como los niños y el adulto mayor.

Es por ello que desde el enfoque legal y poblacional diferencial es importante reconocer la diversidad del ser humano como centro de las políticas y acciones que desde los diferentes sectores e instituciones se formulen y desarrollen, para el mejoramiento de su calidad de vida. Es una estrategia que permite disminuir brechas de desigualdad existentes en algunos de los sectores sociales, etarios y grupos étnicos que habitan la cuidad o el campo, mediante la promoción de la diversidad, la autonomía y el ejercicio de interculturalidad de las ciudadanas y los ciudadanos, a partir del reconocimiento de sus particularidades (Quintero, 2013).

Es fundamental que el adulto mayor dentro de la familia tenga seguridad afectiva y económica, pleno ejercicio de sus derechos humanos y calidad de vida, de manera que tenga el derecho de tener derechos dentro de la familia donde habite (Araque, Beltrán y Pedroza, 2019).

Igualmente es pertinente entender que envejecer implicará menor funcionalidad o independencia especialmente en la etapa de la vejez, por tanto, es necesario promover una sociedad más justa e igualitaria, aunando esfuerzos desde todos los ámbitos con el fin de compartir responsabilidades para acompañar al adulto mayor y favorecerle una vida digna. Por otra parte, se sugiere superar la obligación que imponen las leyes para promover responsabilidad y fomentar que todos los actores sociales implicados en este proceso lo hagan de manera voluntaria y de esta forma aportar soluciones a la vida social y la posibilidad de una vida más humana (Araque y Suárez, 2017)

En definitiva, debemos asumir la responsabilidad social de que la protección y garantía de los derechos humanos son una obligación del Estado, la familia, la academia, pero también de todos y cada uno de nosotros como comunidad ética. 


\section{REFERENCIAS}

Abellán, A. y Esparza, C. (2009). Solidaridad familiar y dependencia entre las personas mayores. Portal Mayores, (99), 2-3.

Agudelo, M. E. (2005). Descripción de la dinámica interna de las familias Monoparentales, simultáneas, extendidas y compuestas del municipio de Medellín, vinculadas al proyecto de prevención temprana de la agresión. Revista Latinoamericana de Ciencias Sociales. Niñez y Juventud, 3(1), 1-19. Recuperado de http://revistaumanizales.cinde.org.co/rlcsnj/ index.php/Revista-Latinoamericana/article/view/311/181

Araque, F., Beltrán, E. y Pedroza, A. (2019). Discapacidad, familia y derechos humanos. Utopía y Praxis Latinoamericana, 24, 206-216. Disponible en https://produccioncientificaluz. org/index.php/utopia/article/view/29776

Araque, F. y Rodríguez, X. (2008). Familias y discurso escolar. Omnia, 14(2), 22-36. Disponible en https://produccioncientificaluz.org/index.php/omnia/article/view/7245/7233

Araque F. y Suárez O. (2017). Reflexiones teóricas y legales del adulto mayor y la discapacidad en Colombia. Jurídicas CUC, 13(1), 97-120. https://doi.org/10.17981/juridcuc.13.1.2017.05

BBC Mundo. (Enero 18, 2018). La soledad, un mal contemporáneo mundial que en Reino Unido ahora es asunto de Estado. $B B C$. [Online]. Disponible en https://www.bbc.com/mundo/ noticias-42723066

Cadavid, I. (1994). La familia como gran organización: concepción sistémica. Revista Universidad Pontifica Bolivariana, 43(138), 117-126. Disponible en https://revistas.upb.edu.co/ index.php/upb/article/view/4648

Cerruti, M. y Bintock, G. (2009). Familias latinoamericanas en transformación: desafíos y demandas para la acción pública. Centro de estudios de población, de Argentina y consultoras de la división de desarrollo social. [LC/L.3100-P]. Santiago: CEPAL. Recuperado de https://repositorio.cepal. org/bitstream/handle/11362/6153/1/S0900608_es.pdf 
Child Trends. (2014). World Family Map. [Online]. Availble from https://www.childtrends.org/wp-content/uploads/2014/04/ WFM-2014-Final-LoRes.pdf

Farne, S. y Rodríguez, D. (2014). Participación de los Adultos Mayores en las Economías de Mercado y del Hogar en Colombia. Bogotá, D.C.: Universidad del Externado.

Gallego, A. (2012). Recuperación crítica de los conceptos de familia, dinámica familiar y sus características. Revista Virtual Universidad Católica del Norte, (35), 326-345.

Gargarella, R. (2019). Las teorías de las justicias después de Rawls. Un breve manual de filosofía política. Barcelona: Paidos.

Helpage International. (2015). Índice Global de Envejecimiento. Agewhatch. [Online]. Recuperado de https://www.helpage. org/silo/files/gawi-2015-resumen-ejecutivo-.pdf

Hernández, J. y Chumaceiro H. A. (2018). Acercamiento histórico a la participación ciudadana en Venezuela: Modelo de relación Estado-Sociedad (1958-2012). Revista de Ciencias Sociales (RCS), 24(2), 56-67. Disponible en https://produccioncientificaluz.org/index.php/rcs/article/view/24819/25349

Huenchuan, S. (ed.) (2018). Envejecimiento, personas mayores y Agenda 2030 para el Desarrollo Sostenible: perspectiva regional y de derechos humanos. (LC/PUB.2018/24-P). Santiago: CEPAL. Recuperado de https://repositorio.cepal.org/ bitstream/handle/11362/44369/1/S1800629_es.pdf

Jaramillo, Á. (Mayo 20, 2019). Más viejos y solos: los hogares unipersonales en Colombia. El Tiempo. [Online]. Disponible en https://www.eltiempo.com/economia/sectores/por-quehay-cada-vez-mas-personas-mayores-viviendo-solas-en-colombia-363954

Landriel, E. (2001). Adultos Mayores y Familia: algunos aspectos de la intervención del Trabajo Social. En, XVII Seminario latinoamericano de escuelas de trabajo social. ALAETS, Lima, Perú. Disponible en http://www.ts.ucr.ac.cr/binarios/ congresos/reg/slets/slets-017-031.pdf 
Lehr, Ú. (1995). Solidaridad entre generaciones: ayuda no estatal para los mayores. El cuidado familiar: posibilidades y limitaciones. En, J. C. Baura, (ed.). Las personas mayores dependientes y el apoyo informal (pp. 145-164). Jaén: Universidad Internacional de Andalucía.

Nieves, M. y Maldonado, C. (ed.). (2011). Las familias latinoamericanas interrogadas. Hacia la articulación del diagnóstico, la legislación y las políticas. [LC/L.3296-P]. Santiago de Chile: CEPAL.

OMS. (2019). Envejecimiento y ciclo de vida. Datos interesantes acerca del envejecimiento. Recuperado de https://www.who. int/ageing/about/facts/es/

OMS. (2017). 10 datos sobre el envejecimiento y la salud. [Online]. Disponible en https://www.who.int/features/factfiles/ ageing/es/

OMS. (2015). Informe mundial sobre el envejecimiento y la salud. Resumen. [Online]. Recuperado de https://apps.who. int/iris/bitstream/handle/10665/186471/WHO_FWC_ ALC_15.01_spa.pdf?sequence=1

OMS. (2011). Informe mundial sobre la discapacidad. [Online]. Recuperado de https://www.who.int/disabilities/world_report/2011/summary_es.pdf?ua=1

ONU. CEPAL. (2017). Envejecimiento provocará caída de la población de América Latina y el Caribe hacia 2060. [Online]. Disponible en https://www.cepal.org/es/comunicados/cepalenvejecimiento-provocara-caida-la-poblacion-america-latina-caribe- 2060

ONU. DAES. (2017). La población mundial aumentará en 1000 millones para 2030. [Online]. Disponible en https://www. un.org/development/desa/es/news/population/world-population-prospects-2017.html

Pachón, X. (2008). La familia en Colombia a lo largo del siglo XX. En, Y.Puyana y M. Himelda (Ed.), Familias, cambios y estrategias (pp. 145-159). Bogotá, D.C.: Universidad Nacional de Colombia. Disponible en http://www.bdigital.unal. edu.co/1363/13/12CAPI11.pdf 
Pedrero, N. L. y Pichardo F. A. (2009). Concepto de funcionalidad, historia clínica geriátrica y evaluación funcional. En, M. González, F. Pichardo y L. García. Geriatría (pp. 32-54). México, D.F.: Mc Graw Hill.

Pedroza A., Suárez-Landazábal, O. y Vargas M. (2018). Adulto mayor: Vida en familia o institucionalización, una reflexión desde la perspectiva global. En, O. Suárez-Landazábal \& M. Silva (Ed.), Envejecimiento y Vejez. Visión interdisciplinaria desde la funcionalidad. (pp. 251-270). EE. UU: Lulu Enterprises.

Pulido, J., Calderón, A. y Arteaga, D. (2018). Imaginarios de constitución de familia que circulan entre la población LGBT y los orientadores escolares del Instituto Educativo Distrital Grancolombiano de la localidad de Bosa. [Tesis de pregrado]. Universidad Pedagógica Nacional de Colombia, Bogotá, D.C., Colombia.

Quintero, A. (2013). La familia según un enfoque de convergencia: diversidad familiar, género y sexualidad. Katharsis, (15), $89-11$.

Reales, A. (2016). La democracia representativa en el marco de los derechos humanos en Colombia. Jurídicas CUC, 12(1), 9-16. http://dx.doi.org/10.17981/juridcuc.12.1.2016.1

República de Colombia. Asamblea Nacional Constituyente. (1991). [Constitución política de Colombia]. Gaceta Constitucional, 1(114).

República de Colombia. Congreso de la República. (27 de noviembre de 2008). Por la cual se dictan normas tendientes a procurar la protección, promoción y defensa de los derechos de los adultos mayores. [Ley 1251]. Diario Oficial: 47.186 .

República de Colombia. DANE. (2018). Resultados censo nacional de población y vivienda 2018. [Online]. Recuperado de https://www.dane.gov.co/files/censo2018/informacion-tecnica/cnpv-2018-presentacion-3ra-entrega.pdf 
República de Colombia. Minsalud. (2018). Sala situacional de personas con discapacidad. [Online]. Recuperado de https://www. minsalud.gov.co/sites/rid/Lists/BibliotecaDigital/RIDE/ DE/PS/sala-situacional-discapacidad-junio-2018.pdf

República de Colombia. Minsalud. (2015). Encuesta Nacional de Salud 2015. [Online]. Recuperado de https://dhsprogram.com/ pubs/pdf/FR334/FR334.pdf

República de Colombia. Minsalud. (2013). Envejecimiento demográfico. Colombia 1951-2020. Dinámica demográfica y estructuras poblacionales. Bogotá, D. C.: Imprenta Nacional. Recuperado de https://www.minsalud.gov.co/sites/rid/Lists/ BibliotecaDigital/RIDE/DE/PS/Envejecimiento-demografico-Colombia-1951-2020.pdf

República de Colombia. Minsalud. Colciencias. (2016). Sabe Colombia 2015: Estudio nacional de salud, bienestar y envejecimiento. [Online]. Recuperado de https://www.minsalud.gov.co/sites/ $\mathrm{rid} /$ Lists/BibliotecaDigital/RIDE/VS/ED/GCFI/ResumenEjecutivo-Encuesta-SABE.pdf

Rodríguez, X., Araque, F. y Salazar, E. (2009). Nociones de Familia en el discurso de Profesionales de Trabajo Social en Espacios Escolares. Revista Alternativas. Cuadernos de Trabajo Social, (16), 91-100. Recuperado de https://rua.ua.es/dspace/bitstream/10045/13546/1/ALT_16_07.pdf

Segovia, M. G. y Torres, E. A. (2011). Funcionalidad del adulto mayor y el cuidado enfermero. Gerokomos, 22(4), 162-166

Sigüenza, W. (2015). Funcionamiento familiar según el Modelo de funcionamiento de Olson. [Tesis de Maestría]. Universidad de Cuenca, Ecuador. Recuperado de http://dspace.ucuenca. edu.ec/jspui/bitstream/123456789/21878/1/TESIS.pdf

Suárez-Landazábal, O. (2018). Envejecimiento y funcionalidad del adulto mayor: reto para el fisioterapeuta. En, M. Silva Pertuz \& O. Suárez-Landazábal (Ed.). El adulto mayor desde la perspectiva de la funcionalidad. (pp. 143-151). EE.UU.: Lulu Enterprises. 
Suárez-Landazábal, O., Batista, L. y Bornacelli, R. (2018). Condición de salud desde la perspectiva de funcionalidad de la población institucionalizada en la ciudad de Barranquilla (Colombia). En, O. Suárez-Landazábal \& M. Silva (Ed.), Envejecimiento y Vejez. Visión interdisciplinaria desde la funcionalidad, (pp. 155-170). EE.UU.: Lulu Enterprises.

Torres, L. E., Ortega, P., Garrido, A. y Reyes, A. G. (2008). Dinámica familiar en familias con hijos e hijas. Revista Intercontinental de Psicología y Educación, 10(2), 31-56.

Olga Suárez Landazábal es Magíster en Investigación y Docencia Universitaria con Formación en Gerontología y Fisioterapeuta. Investigadora de tiempo completo de la Universidad Metropolitana de Barranquilla. Líder del grupo de investigación Educación, Salud y Rehabilitación (EDUSAR) de la Universidad Metropolitana (Colombia).

Francis Araque Barboza es Doctora. en Ciencias Humanas con Magister en Gerencia Pública. Socióloga. Categorizada en Colciencias como Sénior. Docente investigadora del grupo EDUSAR. de la Universidad Metropolitana (Colombia). 